\title{
Relaxation times for Hamiltonian systems
}

\author{
A. Maiocchi* A. Carati*
}

November 8, 2018

\begin{abstract}
Usually, the relaxation times of a gas are estimated in the frame of the Boltzmann equation. In this paper, instead, we deal with the relaxation problem in the frame of the dynamical theory of Hamiltonian systems, in which the definition itself of a relaxation time is an open question. We introduce a lower bound for the relaxation time, and give a general theorem for estimating it. Then we give an application to a concrete model of an interacting gas, in which the lower bound turns out to be of the order of magnitude of the relaxation times observed in dilute gases.
\end{abstract}

\section{Introduction}

The definition and the estimate of relaxation times are problems of central interest when one attempts describing macroscopic systems through microscopic Hamiltonian models.

In the case of gases, these problem are tackled, and solved, in the frame of the Boltzmann equation (see [1]). In such a frame the existence of a relaxation time is somehow obvious, due to the irreversible character of the equation, and the estimate is obtained in terms of the eigenvalues of the linearized equation, about the equilibrium solution. On the other end, Boltzmann equation refers to a reduced description, while we want to tackle the problem considering the complete system. This would require to estimate the time

${ }^{*}$ Università di Milano, Dipartimento di Matematica, Via Saldini 50, 20133 Milano, Italy. E-mail: alberto.maiocchi@unimi.it 
needed for an initial measure in phase space to relax to an asymptotic one. This approach was followed, for example, in [2], for a reversible dissipative model, which should mimic a coupling of the system of interest with two (or more) heat reservoirs.

In the present work, instead, we tackle the problem from the point of view of the dynamical theory of Hamiltonian systems, for systems which are isolated. In this perspective, a partial answer to the problem is given by Kubo's linear response theory [3]. Indeed, such a theory enables one, at least in principle, to compute in microscopic terms the macroscopic transport coefficients, and then, via macroscopic equations, the relaxation time. From our point of view, however, this answer is not completely satisfactory, because it appeals to macroscopic irreversible equations, which should preliminarily be deduced from the microscopic ones.

A related but different approach is followed here, whose main scheme can be sketched as follows.

From linear response theory we take the starting point, namely, the idea of following the time evolution of the probability distribution function in phase space (and not in the reduced $\mu$-space, as in the Boltzmann equation), when a perturbation $-h A(p, q)$ to the original Hamiltonian $H_{0}(p, q)$ is introduced. Still following Kubo, we then choose to concentrate our attention on a particular observable, namely the one conjugated to the perturbing field, in the familiar sense in which pressure is conjugated to volume and magnetization to the magnetic field. As an example, later on in this paper we will deal with the simple case in which the perturbing field is gravity, and the conjugate observable is the height of the center of mass. Then, our attention is addressed at defining and estimating the relaxation time.

In the spirit of the Kubo approach it is natural to say that equilibrium is attained when the time derivative of the distribution function is negligible. The aim of the present paper is indeed to give a lower bound $t_{\text {inf }}$ to the relaxation time, looking at the evolution not of the distribution function itself, but of the time-derivative of the variable conjugated to the perturbation $A$ in the Hamiltonian, which is strictly related to the distribution function (see formula (11) below). It is easily seen that the time-derivative of the conjugate variable is the function $B \stackrel{\text { def }}{=}\left[H_{0}, A\right]$, namely, the time-derivative of $A$ with respect to the flow generated by the full Hamiltonian $H$ (here, $[\cdot, \cdot]$ denotes Poisson bracket), so that this is the quantity on which we will concentrate in this paper. 
Having chosen the relevant function, namely $B$, we make use of the easily established properties (see later) that its expectation vanishes at equilibrium, and that its time-derivative is positive at the initial time. Thus a lower bound $t_{\text {inf }}$ to the relaxation time is provided by the time before which the time-derivative of the expectation of $B$ is proven to be positive.

The problem is then that one should make use of suitable a priori estimates on the dynamics, since an explicit integration of the equations of motion is lacking. This can actually be implemented following the main idea introduced in paper [4, which was concerned with Hamiltonian perturbation theory in the thermodynamic limit. In such a paper, a procedure is given which, for any $\mathcal{L}^{2}$ function $f$ of phase space with respect to the Gibbs measure, allows one to provide an upper bound to $\left\|U_{t} f-f\right\|_{2}$, by knowing an upper bound to $\|[f, H]\|_{2}$. Here, $H$ is the Hamiltonian of the system, and $U_{t}$ the corresponing unitary evolution group.

The estimate of the lower bound $t_{i n f}$ is provided by formula (8) of Theorem 1, which is stated and proved in Section 2. Such a proof is given for an ample class of Hamiltonian systems, which are the ones considered in most rigorous works in Statistical Mechanics (see [5]).

In Section 3 the general theorem is applied to the case of a gas of interacting point-particles enclosed in a cubic box, to which the gravity force is added as a perturbation. To this aim, we give an interesting estimate of the $s$-point correlation function for a gas interacting through a stable and tempered two-body potential, which is here obtained by extending some old results of Bogolyubov et al. [6] and of O. Penrose [7]. The lower bound to the relaxation time thus found turns out to be comparable with the typical relaxation times observed in dilute gases.

Some further comments are given in Section 4.

\section{General Theorem about Relaxation Times}

We consider an isolated Hamiltonian system, with phase space $\mathcal{M}$, and with an invariant measure with respect to the unperturbed Hamiltonian $H_{0}$. One could think that, in principle, one has to take the microcanonical measure, but, in view of the ensemble equivalence for large $N$ (see, for instance, [8]), we will take, instead, the Gibbs measure at inverse temperature $\beta$, i.e., the 
measure with density $\rho_{0}$ given by

$$
\rho_{0}=\frac{1}{Z_{0}} \exp \left(-\beta H_{0}\right)
$$

$Z_{0}$ being the partition function. Suppose at time 0 a perturbation $-h A(p, q)$ is introduced, where $A$ is a given function on phase space and the parameter $h>0$ controls the size of the perturbation. So, at positive times the Hamiltonian is $H_{1}=H_{0}-h A$. The corresponding Gibbs density (at the same $\beta$ ) will be denoted by $\rho_{1}$. Our aim is to find a sensible lower bound for the relaxation time to the final equilibrium with respect to the full Hamiltonian $H_{1}$.

To this end, along the scheme sketched in the Introduction, following Kubo we consider the observable $B$ defined by

$$
B=\left[A, H_{0}\right],
$$

i.e., the time derivative of the perturbation $A$ with respect to the flow generated by the full Hamiltonian $H_{1}$. We then consider the probability density $\rho$, solution of Liouville's equation relative to the total Hamiltonian $H_{1}$ with initial condition $\rho(0)=\rho_{0}$, and look at the evolution of the expectation of $B$, i.e., we look at the quantity

$$
\bar{B}(t)=\int B \rho(t) \mathrm{d} p \mathrm{~d} q
$$

The quantity of interest actually will be its increment

$$
\Delta \bar{B}(t) \stackrel{\text { def }}{=} \bar{B}(t)-\bar{B}(0)
$$

Writing $\rho$ in the form

$$
\rho(t) \stackrel{\text { def }}{=} \rho_{0}+\Delta \rho(t)
$$

one has

$$
\Delta \bar{B}(t)=\int B \Delta \rho(t) \mathrm{d} p \mathrm{~d} q .
$$

We will show that under the familiar conditions which entail reversibility (namely, that both $H_{0}$ and $A$ are even in the momenta), the quantity $\Delta \bar{B}$ vanishes not only (as it is obvious) at time zero, but also at equilibrium with respect to the full Hamiltonian $H_{1}$. This is due to the fact that the 
expectations of $B$ with respect to the Gibbs densities $\rho_{0}$ and $\rho_{1}$ corresponding to the Hamiltonians $H_{0}$ and $H_{1}$, both vanish by symmetry, because $\rho_{0}$ and $\rho_{1}$ are even in the momenta, whereas $B$ is odd. On the other hand, it turns out that $\Delta \bar{B}$ is initially an increasing function of time, since its time-derivative is positive at time 0 , as it will be shown later. Thus, the time-derivative of $\Delta \bar{B}$ has to become negative at some time if equilibrium with respect to the full Hamiltonian has to be attained, and consequently a lower bound to the relaxation time is provided by the time $t_{\text {inf }}$ up to which the time derivative of $\Delta \bar{B}$ is guaranteed to be positive.

We thus define the lower bound $t_{\text {inf }}$ by

$$
t_{\text {inf }} \stackrel{\text { def }}{=} \sup t^{*}
$$

where $t^{*}$ is such that

$$
\frac{\mathrm{d}}{\mathrm{d} t} \Delta \bar{B}(t)>0 \text { for all } 0<t<t^{*}
$$

or $t_{\text {inf }}=+\infty$ if

$$
\frac{\mathrm{d}}{\mathrm{d} t} \Delta \bar{B}(t)>0 \quad \forall t>0 .
$$

Notice that our definition makes sense also for $h=0$, in which case one has $t_{\text {inf }}=0$, as can be seen by formula (13) below.

The problem is then to estimate the rate of growth of $\Delta \bar{B}$. Now, on the one hand, following Kubo we know that $\Delta \bar{B}(t)$ is strictly related to the time-autocorrelation of $B$ (see (13) below). On the other hand, we can make use of the main result obtained in paper [4], in which it was shown how to estimate the time-autocorrelation of $B$ in terms of the Hamiltonian. Indeed, from the main result of that paper one easily obtains the following property: an a priori estimate of the type

$$
\left\|\left[B, H_{0}\right]\right\|_{0} \leq \eta\|B\|_{0},
$$

(with the norm defined below) implies that the time-evolution of $\Delta \bar{B}$ is slow if $\eta$ is small, or, more precisely, that the lower bound $t_{\text {inf }}$ to the relaxation time defined by (3), (4) is inversely proportional to $\eta$.

Here, $\|\cdot\|_{0}$ is the norm on $\mathcal{L}_{0}^{2}(\mathcal{M})$, the Hilbert space of square integrable complex functions on $\mathcal{M}$, with respect to $\rho_{0}$. We will also have to consider the Hilbert space $\mathcal{L}_{1}^{2}(\mathcal{M})$ of the square integrable complex functions with respect to $\rho_{1}$. The corresponding $\mathcal{L}^{2}$-norm will be denoted by $\|\cdot\|_{1}$. 
Under the rather natural condition (5) given below, which ensures the smallness of the "change" of the Gibbs measure induced by the perturbation $-h A$, for a large class of observables it can be proven that the two norms just introduced are asymptotically equivalent as $h \rightarrow 0$. Indeed one has the following lemma, whose proof is deferred to Appendix A.

Lemma 1 Assume there exist $\delta>0$ such that

$$
\int_{\mathcal{M}} \mathrm{d} p \mathrm{~d} q e^{\delta A} \rho_{0}<+\infty \quad \text { and } \quad \int_{\mathcal{M}} \mathrm{d} p \mathrm{~d} q e^{-\delta A} \rho_{0}<+\infty .
$$

Then, for all real functions $f$ on $\mathcal{M}$ satisfying at least one of the conditions

$$
\left\|f^{2}\right\|_{0}<+\infty, \quad\left\|f^{2}\right\|_{1}<+\infty
$$

one has

$$
\|f\|_{1}^{2}-\|f\|_{0}^{2}=o(1), \quad \text { as } h \rightarrow 0 .
$$

We are now able to give an estimate for $t_{\text {inf }}$ in terms of $\eta$, which is provided by the following Theorem 1. It will be seen that some technical hypotheses, namely those given in (7) below, are required just in order that at least one of the conditions (6) of Lemma 1 is satisfied.

Theorem 1 Let the unperturbed Hamiltonian $H_{0}(p, q)$ be even in the momenta and bounded from below, and consider a perturbation - $h A(p, q)$, with $h>0$ and $A$ even in the momenta. Suppose $A$ and $H_{0}$ are such that hypothesis (5) of Lemma 1 is satified. With $B=\left[A, H_{0}\right]$, suppose furthermore that the following technical conditions are satisfied:

$$
\left\|B^{4}\right\|_{0}<+\infty ; \quad\left\|\left[B, H_{0}\right]^{2}\right\|_{0}<+\infty ; \quad\left\|[B, A]^{2}\right\|_{0}<+\infty
$$

Then a lower bound to the relaxation time defined by (3), (4) is given by

$$
t_{i n f} \geq \frac{\sqrt{2}}{\eta}+o(1), \quad \text { as } \quad h \rightarrow 0,
$$

where $\eta$ is such that

$$
\left\|\left[B, H_{0}\right]\right\|_{0}<\eta\|B\|_{0} .
$$


Remark. It may appear that some conditions are too restrictive if the theorem has to be used in the thermodynamic limit, but it turns out that such a difficulty can be overcome. For example, $H_{0}$ was required to have a finite lower bound, call it $D$; however, the result is found not to depend on the value of $D$. So, $D$ can grow with the number $N$ of degrees of freedom, without affecting the validity of the theorem, provided $D$ is finite for any finite $N$. A similar argument also applies to conditions (5) and (7), so the theorem holds for any system, however large it may be. Then, in order to pass to the thermodynamic limit, it suffices to have that $\eta$ tends uniformly to a finite limit as $N$ increases.

Proof of Theorem 1. First of all, we notice that the time evolution of the perturbation $\Delta \rho$ satisfies, by Liouville's equation, the differential equation

$$
\frac{\partial \Delta \rho}{\partial t}=\left[H_{0}-h A, \Delta \rho\right]-h\left[A, \rho_{0}\right],
$$

with $\Delta \rho(0)=0$ as initial condition. Such an equation admits a unique solution in the Hilbert space $\mathcal{L}_{1}^{2}(\mathcal{M})$. Indeed, equation (10) is a linear inhomogeneous first-order differential equation in $\mathcal{L}_{1}^{2}(\mathcal{M})$ of the form

$$
\dot{x}=\hat{\mathcal{O}} x+f,
$$

where the operator $\hat{\mathcal{O}} \stackrel{\text { def }}{=}\left[H_{1}, \cdot\right]$ generates a semigroup of unitary evolution transformations (see for example [9]). Thus, since the second term $h\left[A, \rho_{0}\right]$ at the r.h.s. belongs to $\mathcal{L}_{1}^{2}(\mathcal{M})$, as will be shown below, the solution is known to exist and be unique (see Theorem 3.3, page 104, of [10]). Such a solution is given by a simple adaptation of the variation of constants formula, namely by

$$
\Delta \rho(\mathbf{x}, t)=\beta h \int_{0}^{t} d s B\left(\Phi^{s} \mathbf{x}\right) \rho_{0}\left(\Phi^{s} \mathbf{x}\right),
$$

where $\mathbf{x} \stackrel{\text { def }}{=}(p, q)$ denotes a point of phase space $\mathcal{M}$, and $\Phi^{t}$ the flow generated by $H_{1}$. Notice that, as the initial datum vanishes, one obviously has $\hat{\mathcal{O}} \Delta \rho(0) \in \mathcal{L}_{1}^{2}(\mathcal{M})$.

We show now that $\left[A, \rho_{0}\right] \in \mathcal{L}_{1}^{2}(\mathcal{M})$, too. To this end, we first notice that

$$
\left\|\left[A, \rho_{0}\right]\right\|_{1}=\left\|B \rho_{0}\right\|_{1} \leq \frac{e^{\beta D}}{Z_{0}}\|B\|_{1},
$$


where $D \stackrel{\text { def }}{=} \inf _{p, q} H_{0}$. On the other hand, iterating the Schwarz inequality gives

$$
\|B\|_{0} \leq\left(\left\|B^{2}\right\|_{0}\right)^{\frac{1}{2}} \leq\left(\left\|B^{4}\right\|_{0}\right)^{\frac{1}{4}}<+\infty,
$$

in which the first hypothesis of (7) was used 1 By virtue of such an hypothesis, we can also apply Lemma 1 to $B$ and observe that, for $h$ small enough, $\|B\|_{1}$ is finite. Thus, by (12) it is proved that $\left[A, \rho_{0}\right]$ belongs to $\mathcal{L}_{1}^{2}(\mathcal{M})$.

We now look at the expectation $\bar{B}(t)$ and at its increment $\Delta \bar{B}(t)$. By using (11) for $\Delta \rho$ in (2), one finds for $\Delta \bar{B}(t)$ the expression

$$
\Delta \bar{B}(t)=\beta h \int_{\mathcal{M}} \mathrm{d} \mathbf{x} \int_{0}^{t} d s B\left(\Phi^{s} \mathbf{x}\right) B(\mathbf{x}) \rho_{0}\left(\Phi^{s} \mathbf{x}\right) .
$$

Using the shorthand $f\left(\mathbf{x}_{t}\right)=f\left(\Phi^{-t} \mathbf{x}\right)$, one has then:

$$
\frac{\mathrm{d}}{\mathrm{d} t} \Delta \bar{B}(t)=\beta h \int_{\mathcal{M}} \mathrm{d} \mathbf{x} B\left(\mathbf{x}_{-t}\right) B(\mathbf{x}) \rho_{0}\left(\mathbf{x}_{-t}\right),
$$

or equivalently (due to preservation of Lebesgue measure),

$$
\frac{\mathrm{d}}{\mathrm{d} t} \Delta \bar{B}(t)=\beta h \int_{\mathcal{M}} \mathrm{d} \mathbf{x} B\left(\mathbf{x}_{t}\right) B(\mathbf{x}) \rho_{0}(\mathbf{x}) .
$$

At this point we remark that the integral in (14) could be evaluated in a quite simple way, if there appeared $\rho_{1}$ in place of $\rho_{0}$. Indeed, due to the unitarity of the flow, for any $f$ in $\mathcal{L}_{1}^{2}(\mathcal{M})$ one would have

$$
\int_{\mathcal{M}} \mathrm{d} \mathbf{x} f\left(\mathbf{x}_{t}\right) f(\mathbf{x}) \rho_{1}(\mathbf{x})=\|f\|_{1}^{2}-\frac{1}{2}\left\|f\left(\mathbf{x}_{t}\right)-f(\mathbf{x})\right\|_{1}^{2},
$$

and thus, on account of hypothesis (9) of the theorem, the thesis would follow by using Theorem 1 of [4] (see below).

The rest of the proof is devoted to show that the error made by taking $\rho_{0}$ in place of $\rho_{1}$ is negligible in the limit $h \rightarrow 0$. To this end, we suitably rewrite (14) in the form

$$
\begin{gathered}
\frac{\mathrm{d}}{\mathrm{d} t} \Delta \bar{B}(t)=\beta h\left[\int_{\mathcal{M}} \mathrm{d} \mathbf{x} B\left(\mathbf{x}_{t}\right) B(\mathbf{x}) \rho_{1}(\mathbf{x})-\int_{\mathcal{M}} \mathrm{d} \mathbf{x} B\left(\mathbf{x}_{t}\right) B(\mathbf{x})\left(\rho_{1}(\mathbf{x})-\rho_{0}(\mathbf{x})\right)\right] \\
\geq \beta h\left[\int_{\mathcal{M}} \mathrm{d} \mathbf{x} B\left(\mathbf{x}_{t}\right) B(\mathbf{x}) \rho_{1}(\mathbf{x})-\left|\int_{\mathcal{M}} \mathrm{d} \mathbf{x} B\left(\mathbf{x}_{t}\right) B(\mathbf{x})\left(\rho_{1}(\mathbf{x})-\rho_{0}(\mathbf{x})\right)\right|\right]
\end{gathered}
$$

\footnotetext{
${ }^{1}$ According to the same reasoning, the square of the norm of a function will be bounded from above by the norm of the squared function.
} 
First, we show that the second term at the r.h.s. vanishes as $h \rightarrow 0$. Indeed, by Schwarz's inequality we have

$$
\left|\int_{\mathcal{M}} \mathrm{d} \mathbf{x} B\left(\mathbf{x}_{t}\right) B(\mathbf{x})\left(\rho_{1}(\mathbf{x})-\rho_{0}(\mathbf{x})\right)\right| \leq\left[\int_{\mathcal{M}} \mathrm{d} \mathbf{x}\left(B^{2}\left(\mathbf{x}_{t}\right) B^{2}(\mathbf{x})\right) \rho_{1}(\mathbf{x})\right]^{\frac{1}{2}} \sqrt{\tilde{\gamma}(h)},
$$

where we have defined

$$
\tilde{\gamma}(h) \stackrel{\text { def }}{=} \int_{\mathcal{M}} \mathrm{d} \mathbf{x}\left(\frac{\rho_{0}(\mathbf{x})}{\rho_{1}(\mathbf{x})}-1\right)^{2} \rho_{1}(\mathbf{x}) .
$$

This function coincides with the one defined by (50) in Appendix A. As there shown, one has $\tilde{\gamma}(h) \rightarrow 0$ as $h \rightarrow 0$. We then make use of (15), by replacing $B^{2}$ for $f$ and neglecting the negative term, in order to find an upper bound to the r.h.s. of (17): one has, in fact,

$$
\left[\int_{\mathcal{M}} \mathrm{d} \mathbf{x}\left(B^{2}\left(\mathbf{x}_{t}\right) B^{2}(\mathbf{x})\right) \rho_{1}(\mathbf{x})\right]^{\frac{1}{2}} \leq\left\|B^{2}\right\|_{1} .
$$

In order to show that $\left\|B^{2}\right\|_{1}$ is finite, we use Lemma 1, whose hypotheses are satisfied owing to the first inequality of (7). Thus, as $\tilde{\gamma}(h) \rightarrow 0$ for $h \rightarrow 0$, one gets

$$
\left|\int_{\mathcal{M}} \mathrm{d} \mathbf{x} B\left(\mathbf{x}_{t}\right) B(\mathbf{x})\left(\rho_{1}(\mathbf{x})-\rho_{0}(\mathbf{x})\right)\right|=o(1) \quad \text { as } \quad h \rightarrow 0 .
$$

We then come to the first term at the r.h.s. of (16), which, using (15) again, can be estimated as

$$
\int_{\mathcal{M}} \mathrm{d} \mathbf{x} B\left(\mathbf{x}_{t}\right) B(\mathbf{x}) \rho_{1}(\mathbf{x})=\|B\|_{1}^{2}-\frac{1}{2}\left\|B\left(\mathbf{x}_{t}\right)-B(\mathbf{x})\right\|_{1}^{2} .
$$

We now make use of Theorem 1 of [4], which ensures that, if

$$
\left\|\left[B, H_{1}\right]\right\|_{1}<\tilde{\eta}\|B\|_{1}
$$

is satisfied, then one has

$$
\left\|B\left(\mathbf{x}_{t}\right)-B(\mathbf{x})\right\|_{1}<\tilde{\eta} t\|B\|_{1} .
$$


Now, to give an estimate for $\tilde{\eta}$, we notice that the following inequalities hold as $h \rightarrow 0$ :

$$
\begin{aligned}
\left\|\left[B, H_{1}\right]\right\|_{1} \leq & \left\|\left[B, H_{0}\right]\right\|_{1}+h\|[B, A]\|_{1} \\
\leq & \left(\left\|\left[B, H_{0}\right]\right\|_{0}^{2}+o(1)\right)^{\frac{1}{2}} \\
& +h\left(\|[B, A]\|_{0}^{2}+o(1)\right)^{\frac{1}{2}} .
\end{aligned}
$$

Here, in the first line the triangle inequality was used, while the second line is a consequence of Lemma 1, the hypotheses of which are satisfied by virtue of the second and the third inequalities in (7). Hence, by hypothesis (9) we obtain

$$
\begin{aligned}
\left\|\left[B, H_{1}\right]\right\|_{1} & \leq\left\|\left[B, H_{0}\right]\right\|_{0}+o(1)\|B\|_{1} \\
& <\eta\|B\|_{0}+o(1)\|B\|_{1} \\
& \leq(\eta+o(1))\|B\|_{1}
\end{aligned}
$$

so that $\tilde{\eta}=\eta+o(1)$.

Eventually, by replacing in (16) the estimates (18) and (19), one has

$$
\frac{\mathrm{d}}{\mathrm{d} t} \Delta \bar{B}(t) \geq \beta h\left(1-\frac{\eta^{2} t^{2}}{2}-o(1)-o(1) \cdot t^{2}\right)\|B\|_{1}^{2} .
$$

Therefore, in the limit as $h \rightarrow 0$, the time derivative of $\Delta \bar{B}(t)$ remains positive for

$$
t<\frac{\sqrt{2}}{\eta}+o(1)
$$

and this completes the proof.

Q.E.D.

\section{A Gas in a Gravitational Field}

We study now a gas of $N$ interacting particles enclosed in a tridimensional box of side $L$ and total volume $V=L^{3}$. Our aim is to show that Theorem 1 holds if we take as a simple example of perturbation the force of gravity, in which case the conjugated variable will be the displacement on the vertical axis of the center of mass of the system. 
For what concerns the interaction of the particles with the walls, due to the form of the conjugated variable it turns out that only the interaction with the horizontal walls will matter. Thus we limit ourselves to choose a particular form for the interaction potential with the horizontal walls. The unperturbed Hamiltonian $H_{0}$ is then

$$
H_{0} \stackrel{\text { def }}{=} \sum_{j=1}^{N} \frac{\mathbf{p}_{j}^{2}}{2}+U_{N}\left(\mathbf{q}_{1}, \ldots, \mathbf{q}_{N}\right)
$$

where $p_{j}^{\alpha} \in(-\infty,+\infty), q_{j}^{\alpha} \in(-L / 2, L / 2), \alpha=1,2,3, q_{j}^{3}=z_{j}$, and $U_{N}$ denotes the potential energy of the system, which we take of the form

$$
U_{N} \stackrel{\text { def }}{=} \sum_{1 \leq i<j \leq N} \Phi\left(\mathbf{q}_{i}-\mathbf{q}_{j}\right)+\sum_{i=1}^{N} f\left(z_{i}\right)
$$

where $\Phi$ represents the mutual interaction potential between the particles and $f$ the interaction with the horizontal walls.

The choice of the possible forms of $\Phi$ and $f$ is restricted by some technical conditions, which guarantee the existence of a suitable upper bound to the configuration integrals. In fact, the main difficulty which is encountered in applying Theorem 1 to the present case is the estimate of the distribution function for $s$ particles, often called the $s$-point correlation function. We thus define

$$
F_{s}^{(N)}\left(\mathbf{q}_{1}, \ldots, \mathbf{q}_{s}\right) \stackrel{\text { def }}{=} V^{s} \int_{V} \mathrm{~d}^{3} \mathbf{q}_{s+1} \ldots \int_{V} \mathrm{~d}^{3} \mathbf{q}_{N} D_{N}\left(\mathbf{q}_{1}, \ldots, \mathbf{q}_{N}\right),
$$

where

$$
D_{N}\left(\mathbf{q}_{1}, \ldots, \mathbf{q}_{N}\right) \stackrel{\text { def }}{=} \frac{1}{Q_{N}} \exp \left[-\beta U_{N}\left(\mathbf{q}_{1}, \ldots, \mathbf{q}_{N}\right)\right]
$$

and

$$
Q_{N} \stackrel{\text { def }}{=} \int_{V} \mathrm{~d}^{3} \mathbf{q}_{1} \ldots \int_{V} \mathrm{~d}^{3} \mathbf{q}_{N} \exp \left[-\beta U_{N}\left(\mathbf{q}_{1}, \ldots, \mathbf{q}_{N}\right)\right] .
$$

For sufficiently low densities $\rho=N / V$, one can prove a useful lemma which relates $F_{s}^{(N)}$ to

$$
n_{s}\left(\mathbf{q}_{1}, \ldots, \mathbf{q}_{s}\right) \stackrel{\text { def }}{=} \exp \left[-\beta U_{s}\left(\mathbf{q}_{1}, \ldots, \mathbf{q}_{s}\right)\right],
$$

under suitable conditions for the potentials $\Phi$ and $f$. We consider $\Phi$ to be a stable and tempered potential in the familiar sense (see, for example, [5]). Then, in Appendix $\mathrm{B}$ we prove the following 
Lemma 2 Let $\beta$ be fixed. Let $\Phi$ be a stable potential, i.e. suppose that exist $b>0$ such that

$$
\sum_{1 \leq i<j \leq s} \beta \Phi\left(\mathbf{q}_{i}-\mathbf{q}_{j}\right) \geq-s b ;
$$

furthermore, assume that $\Phi$ is tempered and that $V$ is so large that one has

$$
I \stackrel{\text { def }}{=} \int_{\mathbb{R}^{3}}\left|e^{-\beta \Phi(\mathbf{r})}-1\right| \mathrm{d}^{3} \mathbf{r}<\frac{e-2}{2(e+1)} V .
$$

Let $f$ be continuous on the open interval $(-L / 2, L / 2)$, nonnegative and such that

$$
\tilde{L} \stackrel{\text { def }}{=} \int_{-\frac{L}{2}}^{\frac{L}{2}} \mathrm{~d} z e^{-\beta f(z)}>\frac{L}{2} .
$$

Then, for all densities $\rho$ satisfying

$$
\rho<\min \left[\frac{1}{I}\left(\frac{\tilde{L}}{L}-\frac{1}{2}\right), \frac{1}{4 e^{2 b+1} I}\right],
$$

the following inequality holds.

$$
F_{s}^{(N)}\left(\mathbf{q}_{1}, \ldots, \mathbf{q}_{s}\right)<\frac{2^{s} \sqrt{e}}{\sqrt{e}-1} \exp \left(\frac{s I_{s}}{2 I}\right) n_{s}\left(\mathbf{q}_{1}, \ldots, \mathbf{q}_{s}\right),
$$

where $I_{1}=I$ and, for $s \geq 2$,

$$
I_{s} \stackrel{\text { def }}{=} \int_{\mathbb{R}^{3}} \max \left\{\left(1-e^{-\beta \Phi(\mathbf{r})}\right), e^{2 s b}\left(1-e^{\beta \Phi(\mathbf{r})}\right)\right\} \mathrm{d}^{3} \mathbf{r} .
$$

In order to use such a lemma and have, at the same time, a physically relevant model without complicating too much the computations, we take $\Phi$ and $f$ equal to the repulsive part of the Lennard-Jones potential, namely, given by

$$
\begin{aligned}
\Phi(\mathbf{x}-\mathbf{y}) & \stackrel{\text { def }}{=} \frac{\varepsilon}{\|\mathbf{x}-\mathbf{y}\|^{12}}, \\
f(z) & \stackrel{\text { def }}{=} \delta\left[\left(\frac{1}{z+\frac{L}{2}}\right)^{12}+\left(\frac{1}{z-\frac{L}{2}}\right)^{12}\right],
\end{aligned}
$$

where $\varepsilon$ and $\delta$ are positive parameters, chosen in a convenient way (see hypothesis (37) of Theorem 2). 
According to the general scheme previously discussed, we add at time $t=0$ a perturbation $-h A$, and for the observable $A$ we make the choice

$$
A \stackrel{\text { def }}{=} \sum_{j=1}^{N} z_{j}
$$

We then have

Theorem 2 Let $H_{0}$ and $A$ be given by (23) and (36) respectively. Let the parameters $\varepsilon, \delta$ and $L$ in (35) be such that

$$
(\beta \varepsilon)^{\frac{1}{12}}<L / 3 \text { and }(\beta \delta)^{\frac{1}{12}}<L / 5
$$

where $\beta$ is the inverse temperature. Then, for all densities $\rho$ which satisfy

$$
\rho<\frac{1.5 \cdot 10^{-2}}{(\beta \varepsilon)^{\frac{1}{4}}},
$$

one has the estimate $t_{\text {inf }} \geq t_{0}+o(1)$, as $h \rightarrow 0$, with

$$
t_{0}=\frac{1}{c}(\beta \delta)^{\frac{1}{24}} \sqrt{L \beta}
$$

and

$$
c \stackrel{\text { def }}{=} 2\left(\frac{13 e}{\sqrt{e}-1} \int_{0}^{+\infty} u^{\frac{1}{12}} e^{-u} \mathrm{~d} u\right)^{\frac{1}{2}} \approx 14.5 .
$$

Remark 1. The theorem is stated for a dimensionless Hamiltonian. By inserting the proper dimensions, condition $(\beta \delta)^{\frac{1}{12}}<L / 5$ becomes $(\beta \delta)^{\frac{1}{12}} \sigma<$ $L / 5$, where $\sigma$ is the charachteristic parameter for the range of the LennardJones potential. This just expresses the requirement that the interactions with the walls have a range which is negligible with respect to $L$. Notice that we inserted the factor $1 / 5$ at the r.h.s. just in order to fix a numerical value for $c$, but any other reasonable choice would not affect the result. A similar reasoning holds for the other requirement in (37). Notice, however, that the value of $t_{0}$ does not depend on the interaction potentia $\sqrt[2]{2} \Phi$, which affects only the density up to which the result is valid, through formula (38).

\footnotetext{
${ }^{2}$ This is true for every potential $\Phi$ for which Lemma 2 can be applied (see the discussion concerning (42) and (43) in the proof).
} 
Remark 2. The time $t_{0}$, once the correct dimensional constants have been introduced, becomes

$$
t_{0}=\frac{1}{c}(\beta \delta)^{\frac{1}{24}} \sqrt{\beta m L \sigma},
$$

where $m$ is the mass (of molecular order) of each particle. Therefore, for macroscopic systems in which $L$ is of the order of magnitude of $1 \mathrm{~m}$, while $\sigma \approx 10^{-10} \mathrm{~m}$, at room temperature one gets $t_{0} \approx 10^{-8} \mathrm{~s}$, a value which is of the order of magnitude of the typical relaxation times measured in gases. In the same way, condition (38) is proved to hold for ordinary densities, namely, of the order of magnitude of $10^{24} \mathrm{~m}^{-3}$. Further comments will be given in the next section.

Proof. The proof consists in showing that the hypotheses of Theorem 1 hold, with

$$
\eta=\frac{\sqrt{2} c}{(\beta \delta)^{\frac{1}{24}} \sqrt{L \beta}} .
$$

In the first place, (5) is satisfied for any $\delta$, because it involves integrals of continuous functions over a compact, and the integral over the $\mathbf{p}$ coordinates is equal to 1 .

As far as the other hypotheses are concerned, we first notice that in the present case one has

$$
B=\sum_{j=1}^{N} p_{j}^{z} .
$$

The integral over the momenta of any power of $B$ can be easily turned into a combination of terms of the form

$$
\int_{-\infty}^{+\infty} \mathrm{d} p p^{n} \exp \left(\frac{-\beta p^{2}}{2}\right),
$$

which are finite for any $n$, thus proving the first of (7). In particular, one has

$$
\|B\|_{0}=\sqrt{\frac{2 N}{\beta}} .
$$

Clearly, one also has

$$
[B, A]=N,
$$


and hence the third of (17) holds. We then compute $\left\|\left[B, H_{0}\right]\right\|_{0}$. One has

$$
\left[B, H_{0}\right]=-\sum_{j=1}^{N} f^{\prime}\left(z_{j}\right)
$$

with

$$
f^{\prime}\left(z_{j}\right)=-12 \delta \sum_{j=1}^{N}\left[\left(\frac{1}{z_{j}+\frac{L}{2}}\right)^{13}+\left(\frac{1}{z_{j}-\frac{L}{2}}\right)^{13}\right]
$$

since the contribution due to the pair interaction is the $z$-component of the sum of all the internal forces, and consequently it vanishes. Function (42), and its powers too, are actually singular at some point in phase space, but they diverge there as a power, while the density $\rho_{0}$ vanishes as an exponential, making the norm finite. So the second of (17) is proved, as well.

There finally remains the task of providing an estimate for the quantity $\eta$, which is an upper bound for the ratio $\|\left[B, H_{0}\left\|_{0} /\right\| B \|_{0}\right.$. To this end, from (42) we get

$$
\left[B, H_{0}\right]^{2}=\sum_{j, l=1}^{N} f^{\prime}\left(z_{j}\right) f^{\prime}\left(z_{l}\right)
$$

and we have to estimate its $\rho_{0}$ norm.

It is convenient to integrate by parts: we use the equality

$$
\begin{aligned}
f^{\prime}\left(z_{j}\right) f^{\prime}\left(z_{l}\right) e^{-\beta U_{N}}= & \frac{1}{\beta^{2}} \frac{\mathrm{d}}{\mathrm{d} z_{l}} \frac{\mathrm{d}}{\mathrm{d} z_{j}} e^{-\beta U_{N}}-\frac{1}{\beta} \frac{\mathrm{d}}{\mathrm{d} z_{l}}\left(F_{j}^{z} e^{-\beta U_{N}}\right)-F_{l}^{z} f^{\prime}\left(z_{j}\right) e^{-\beta U_{N}} \\
& +\frac{\delta_{j l}}{\beta} f^{\prime \prime}\left(z_{j}\right) e^{-\beta U_{N}}
\end{aligned}
$$

where $\delta_{j l}$ is the Krönecker delta and by $F_{j}^{z}$ we denote the $z$ component of the force exerted on the $j$-th particle by the other particles, i. e.

$$
F_{j}^{z} \stackrel{\text { def }}{=}-\frac{\mathrm{d}}{\mathrm{d} z_{j}} \sum_{1 \leq i<k \leq N} \Phi\left(\mathbf{q}_{i}-\mathbf{q}_{k}\right)
$$

We observe that the first term at the r.h.s. of (43) has a vanishing inegral, being the derivative of a function which vanishes at the boundaries. Moreover, for what concerns the second term, we point out that the quantity $\sum_{j} F_{j}^{z}$ vanishes, being the $z$ component of the sum of all the internal forces. 
The same remark is in order for the sum over $l$ of the terms in the third place. The only term left is thus the fourth one, which we write in the form $\delta_{j l}$ times the function

$$
\frac{1}{\beta} f^{\prime \prime}\left(z_{j}\right) e^{-\beta U_{N}}=\frac{156 \delta}{\beta}\left[\left(\frac{1}{z_{j}+\frac{L}{2}}\right)^{14}+\left(\frac{1}{z_{j}-\frac{L}{2}}\right)^{14}\right] e^{-\beta U_{N}} .
$$

Therefore, we have to compute $N$ identical integrals of the latter quantity, depending only on one coordinate.

We remark here that conditions (37), (38) are sufficient to ensure that Lemma 2 can be used. In fact, our $\Phi$ is certainly stable, tempered and nonnegative, while $f$ is continuous on $(-L / 2, L / 2)$; furthermore, it is easy to verify the remaining hypotheses, on account of a simple integration for (30) and of numerical computations of the integrals appearing in (31) and (32).

Making use of Lemma 2 for $F_{1}^{(N)}$ one finds that the modulus of the integral of (44), which is independent of $j$, is smaller than

$$
\frac{312 e \delta}{(\sqrt{e}-1) \beta L} \int_{-\frac{L}{2}}^{\frac{L}{2}} \mathrm{~d} z\left[\left(\frac{1}{z+\frac{L}{2}}\right)^{14}+\left(\frac{1}{z-\frac{L}{2}}\right)^{14}\right] e^{-\beta f(z)}
$$

The two terms in the integral (45) are identical, due to symmetry. Each of them is bounded from above on account of the inequality

$$
\begin{aligned}
\int_{-\frac{L}{2}}^{\frac{L}{2}} \mathrm{~d} z\left(\frac{1}{z+\frac{L}{2}}\right)^{14} e^{-\beta f(z)} & \leq \int_{0}^{L} \mathrm{~d} z \frac{1}{z^{14}} \exp \left(-\frac{\beta \delta}{z^{12}}\right) \\
& \leq \frac{1}{12(\beta \delta)^{\frac{13}{12}}} \int_{0}^{+\infty} u^{\frac{1}{12}} e^{-u} \mathrm{~d} u
\end{aligned}
$$

In conclusion, we can infer that

$$
\left\|\left[B, H_{0}\right]\right\|_{0}^{2}<\frac{N c^{2}}{\beta^{\frac{25}{12}} \delta^{\frac{1}{12}}},
$$

and a quick comparison with (41) shows that one has

$$
\left\|\left[B, H_{0}\right]\right\|_{0}<\frac{\sqrt{2} c}{(\beta \delta)^{\frac{1}{24}} \sqrt{L \beta}}\|B\|_{0} .
$$

This relation, on account of Theorem 1, leads to formula (39).

Q.E.D. 


\section{Conclusions}

We have provided by Theorem 1 a lower bound to the relaxation times in Hamiltonian systems, and shown that in the case of an interacting gas enclosed in a box such an estimate is of the order of magnitude of the typical relaxation times measured in gases.

This fact seems to indicate that the interactions with the walls, which we have considered in the present work, might have sensible effects even when one is interested in investigating relaxations of observables related to internal interactions.

We now add some comments concerning possible further developments.

The first point concerns the hypothesis made in Theorem 1, that $H_{0}$ and $A$ are even in the momenta, which actually is not at all essential. Indeed, if such an hypothesis is not satisfied, it suffices to define $t_{\text {inf }}$ in a different way, namely, as the time up to which the time-derivative of $\Delta \bar{B}$ remains larger than, for example, $\frac{1}{2} \frac{\mathrm{d}}{\mathrm{d} t} \Delta \bar{B}(0)$. This way, by inequality (22) one could prove Theorem 1, except for setting $1 / \eta$ in place of $\sqrt{2} / \eta$ in (8). We decided to deal with the case of reversible Hamiltonians just because it is a very important one; furthermore, in such a case the relaxation time can be defined with no reference to arbitrary features, as the factor $1 / 2$ introduced above.

As a more interesting fact, we are confident that our line of reasoning may be extended to the case of perturbations of a finite size $h$, because this would just entail to consider the norms in $\mathcal{L}_{1}^{2}(\mathcal{M})$ rather than in $\mathcal{L}_{0}^{2}(\mathcal{M})$. Indeed, if we substitute $\left[B, H_{1}\right]$ for $\left[B, H_{0}\right]$ in hypothesis (9) and use there the norm $\|\cdot\|_{1}$ instead of $\|\cdot\|_{0}$, we can directly set $\tilde{\eta}=\eta$ in (20) and there is no need of deducing (21), so that the second and third conditions in (7) are no more required. Moreover, the first condition in (7) can be replaced by the condition that $\left\|B^{2}\right\|_{1}$ is finite, which makes trivial the proof that $\left[A, H_{0}\right]$ is in $\mathcal{L}_{1}^{2}(\mathcal{M})$.

The really open problem that remains in order to implement an extension to the case of finite $h$, at least for macroscopic systems, is the estimate of the difference of the two norms in (17). The estimate which appears in such a formula has the serious flaw of increasing exponentially with the number $N$ of particles. This occurs because the upper bound provided there, which is an immediate consequence of the Schwarz inequality, is valid for all functions in $\mathcal{L}^{2}$. A way to improve such an estimate would be to restrict oneself to perturbations having some suitable characteristic features.

In particular, the work [11] of Lanford seems to suggest a good starting 
point. There it is pointed out that the only observables of interest in describing a macroscopic system are the ones he calls finite range observables 3 namely, observables which are sums of terms depending only on the position of a finite number of particles. The difference of the two norms in question can then be evaluated for each term and this should lead to an estimate which doesn't increase too much with $N$. We think that, if one limits oneself to considering a smaller class of functions, there is a good chance that the problem of the number of degrees of freedom is overcome, and that some results are obtained also for the case of perturbations of finite size. These interesting investigations are left for possible future works.

Acknowledgements. We thank very much Professor C. Cercignani and L. Galgani for useful comments and discussions.

\section{A Proof of Lemma 1}

We take as starting point the obvious equality

$$
\int_{\mathcal{M}} \mathrm{d} p \mathrm{~d} q f^{2} \rho_{1}=\int_{\mathcal{M}} d p d q f^{2} \rho_{0}+\int_{\mathcal{M}} \mathrm{d} p \mathrm{~d} q f^{2}\left(\rho_{1}-\rho_{0}\right)
$$

which, by the Schwarz inequality, gives

$$
\left|\int_{\mathcal{M}} \mathrm{d} p \mathrm{~d} q f^{2}\left(\rho_{1}-\rho_{0}\right)\right| \leq\left(\int_{\mathcal{M}} \mathrm{d} p \mathrm{~d} q f^{4} \rho_{0}\right)^{\frac{1}{2}} \sqrt{\gamma(h)}
$$

with

$$
\gamma(h) \stackrel{\text { def }}{=} \int_{\mathcal{M}} \mathrm{d} p \mathrm{~d} q\left(\frac{\rho_{1}}{\rho_{0}}-1\right)^{2} \rho_{0} .
$$

One can also write

$$
\gamma(h)=\frac{\int_{\mathcal{M}} \mathrm{d} p \mathrm{~d} q e^{2 h \beta A} \rho_{0}}{\left(\int_{\mathcal{M}} \mathrm{d} p \mathrm{~d} q e^{h \beta A} \rho_{0}\right)^{2}}-1,
$$

as is seen by expanding the square and using the fact that $\rho_{0}$ and $\rho_{1}$ are the densities of the Gibbs measures corresponding to $H_{0}$ and $H_{1}$, respectively. It

\footnotetext{
${ }^{3}$ As a matter of fact, he explains that this definition is chosen to make things simpler and is too restrictive. He gives also a reference to Ruelle's book [5] in which it is shown how to deal with a broader class of observables, which represents the class of real interest.
} 
is also of interest to provide an upper bound to the l.h.s. of (48) in terms of $\rho_{1}$ rather than of $\rho_{0}$. Indeed, one has

$$
\left|\int_{\mathcal{M}} \mathrm{d} p \mathrm{~d} q f^{2}\left(\rho_{1}-\rho_{0}\right)\right| \leq\left(\int_{\mathcal{M}} \mathrm{d} p \mathrm{~d} q f^{4} \rho_{1}\right)^{\frac{1}{2}} \sqrt{\tilde{\gamma}(h)}
$$

where, in a way similar to (49), one gets

$$
\begin{aligned}
\tilde{\gamma}(h) \stackrel{\text { def }}{=} \int_{\mathcal{M}} \mathrm{d} p \mathrm{~d} q\left(\frac{\rho_{0}}{\rho_{1}}-1\right)^{2} \rho_{1} \\
=\left(\int_{\mathcal{M}} \mathrm{d} p \mathrm{~d} q e^{h \beta A} \rho_{0}\right)\left(\int_{\mathcal{M}} \mathrm{d} p \mathrm{~d} q e^{-h \beta A} \rho_{0}\right)-1 .
\end{aligned}
$$

Now, we observe that the functions $\gamma(h)$ and $\tilde{\gamma}(h)$ can take arbitrarily small values as $h$ goes to 0 , if (5) is satisfied. Indeed, by their definitions, they are always nonnegative quantities. Thus, in order to show, for example, that $\gamma(h)<\varepsilon$ for any fixed positive $\varepsilon$, it will suffice that one has

$$
\frac{\int_{\mathcal{M}} \mathrm{d} p \mathrm{~d} q e^{2 h \beta A} \rho_{0}}{\left(\int_{\mathcal{M}} \mathrm{d} p \mathrm{~d} q e^{h \beta A} \rho_{0}\right)^{2}}<1+\varepsilon
$$

To this end, let us note that

$$
\frac{1}{\int_{\mathcal{M}} \mathrm{d} p \mathrm{~d} q e^{h \beta A} \rho_{0}} \leq \int_{\mathcal{M}} \mathrm{d} p \mathrm{~d} q e^{-h \beta A}
$$

according to the Schwarz inequality. We combine this estimate with the Hölder inequality, on whose account, if $h<\frac{\delta}{c \beta}$ and condition (5) holds, one has

$$
\begin{aligned}
\int_{\mathcal{M}} \mathrm{d} p \mathrm{~d} q e^{ \pm \operatorname{ch} \beta A} \rho_{0} & \leq\left(\int_{\mathcal{M}} \mathrm{d} p \mathrm{~d} q e^{ \pm \delta A} \rho_{0}\right)^{\frac{c h \beta}{\delta}}\left(\int_{\mathcal{M}} \mathrm{d} p \mathrm{~d} q \rho_{0}\right)^{\left(1-\frac{c h \beta}{\delta}\right)} \\
& \leq K^{\frac{c h \beta}{\delta}}
\end{aligned}
$$

for some $K>0$, and we eventually obtain

$$
\begin{aligned}
\frac{\int_{\mathcal{M}} \mathrm{d} p \mathrm{~d} q e^{2 h \beta A} \rho_{0}}{\left(\int_{\mathcal{M}} \mathrm{d} p \mathrm{~d} q e^{h \beta A} \rho_{0}\right)^{2}} & \leq\left(\int_{\mathcal{M}} \mathrm{d} p \mathrm{~d} q e^{2 h \beta A} \rho_{0}\right)\left(\int_{\mathcal{M}} \mathrm{d} p \mathrm{~d} q e^{-h \beta A} \rho_{0}\right)^{2} \\
& \leq K^{\frac{4 h \beta}{\delta}} \rightarrow 1 \text { as } h \rightarrow 0
\end{aligned}
$$


The immediate consequence is that, if we take $h<\min \left(\frac{\delta}{2 \beta}, \frac{\delta \log (1+\varepsilon)}{4 \beta \log K}\right)$, then $\gamma(h)$ is less than $\varepsilon$. An analogous argument, still based on inequality (50), ensures that $\tilde{\gamma}(h)$ takes arbitrarily small values as $h$ goes to 0 , too. Hence, the difference between $\|f\|_{0}^{2}$ and $\|f\|_{1}^{2}$ vanishes with $h$, provided $f^{2}$ belongs to $\mathcal{L}_{0}^{2}(\mathcal{M})$ or $\mathcal{L}_{1}^{2}(\mathcal{M})$.

Q.E.D.

\section{B Proof of Lemma 2}

The lemma is proved by using the results that were obtained in [6] in deducing the Mayer-Montroll equation.

A relevant difference from the classical works in this field is that here we have to deal with an external field, too. In order to connect the computations with the ones used in the absence of such a field, we change the coordinates from $\mathbf{q}_{j}$ to $\tilde{\mathbf{q}}_{j}$, according to

$$
\tilde{q}_{j}^{1}=q_{j}^{1}, \quad \tilde{q}_{j}^{2}=q_{j}^{2}, \quad \tilde{z}_{j}=\int_{-L / 2}^{z_{j}} \mathrm{~d} x e^{-\beta f(x)}
$$

This change of coordinates is well defined, because $f$ is continuous and the following inequality holds:

$$
\frac{\mathrm{d} \tilde{z}_{j}}{\mathrm{~d} z_{j}}>0 \quad \forall z_{j} \in\left(-\frac{L}{2}, \frac{L}{2}\right) .
$$

The volume differential, thus, changes in accordance with

$$
e^{-\beta f\left(z_{j}\right)} \mathrm{d}^{3} \mathbf{q}_{j}=\mathrm{d}^{3} \tilde{\mathbf{q}}_{j},
$$

making the external field disappear in the integration; we denote by $\tilde{V}$ the domain of integration which takes the place of $V$. We must also notice that the pair potential $\Phi\left(\mathbf{q}_{i}-\mathbf{q}_{j}\right)$ is replaced by

$$
\tilde{\Phi}\left(\tilde{\mathbf{q}}_{i}, \tilde{\mathbf{q}}_{j}\right) \stackrel{\text { def }}{=} \Phi\left(\tilde{q}_{i}^{1}-\tilde{q}_{j}^{1}, \tilde{q}_{i}^{2}-\tilde{q}_{j}^{2}, z_{i}\left(\tilde{z}_{i}\right)-z_{j}\left(\tilde{z}_{j}\right)\right)
$$

which is a function of the coordinates of both particles, and not only of their difference, as was the case for $\Phi$, but it is still symmetric under the exchange of $\tilde{\mathbf{q}}_{i}$ and $\tilde{\mathbf{q}}_{j}$. 
Furthermore, we point out here that we must consider a different distribution function in the modified phase space. Such a function, which we call $\tilde{D}_{N}$, is chosen by asking that it preserves the volume element under the change of coordinates from $\mathbf{q}_{i}$ to $\tilde{\mathbf{q}}_{i}$, i. e. that it fulfills the requirement

$$
\tilde{D}_{N}\left(\tilde{\mathbf{q}}_{1}, \ldots, \tilde{\mathbf{q}}_{N}\right) \mathrm{d}^{3} \tilde{\mathbf{q}}_{1} \ldots \mathrm{d}^{3} \tilde{\mathbf{q}}_{N}=D_{N}\left(\mathbf{q}_{1}, \ldots, \mathbf{q}_{N}\right) \mathrm{d}^{3} \mathbf{q}_{1} \ldots \mathrm{d}^{3} \mathbf{q}_{N},
$$

if $\tilde{\mathbf{q}}_{i}=\tilde{\mathbf{q}}_{i}\left(\mathbf{q}_{i}\right)$ for $i=1, \ldots, N$. It is apparent that one has

$$
\tilde{D}_{N}\left(\tilde{\mathbf{q}}_{1}, \ldots, \tilde{\mathbf{q}}_{N}\right)=\frac{1}{Q_{N}} \exp \left[-\beta \sum_{1 \leq i<j \leq N} \tilde{\Phi}\left(\tilde{\mathbf{q}}_{i}, \tilde{\mathbf{q}}_{j}\right)\right] .
$$

Accordingly, we will study the modified $s$-point correlation $\tilde{F}_{s}^{(N)}$, defined in a way similar to (25), replacing $D_{N}$ with $\tilde{D}_{N}$ and $\mathbf{q}$ with $\tilde{\mathbf{q}}$. The relation with the function $F_{s}^{(N)}$, which is the one we want to bound from above, would be

$$
F_{s}^{(N)}\left(\mathbf{q}_{1}, \ldots, \mathbf{q}_{s}\right)=\tilde{F}_{s}^{(N)}\left(\tilde{\mathbf{q}}_{1}\left(\mathbf{q}_{1}\right), \ldots, \tilde{\mathbf{q}}_{s}\left(\mathbf{q}_{s}\right)\right) \exp \left(-\beta \sum_{i=1}^{s} f\left(z_{i}\right)\right)
$$

We can now repeat the deduction of the Mayer-Montroll equations in these new coordinates, by writing

$$
\begin{aligned}
\tilde{F}_{s}^{(N)}\left(\tilde{\mathbf{q}}_{1}, \ldots, \tilde{\mathbf{q}}_{s}\right)= & \frac{V^{s} Q_{N-s}}{Q_{N}}\left[\int_{\tilde{V}} \mathrm{~d}^{3} \tilde{\mathbf{q}}_{1}^{*} \ldots \int_{\tilde{V}} \mathrm{~d}^{3} \tilde{\mathbf{q}}_{N-s}^{*} \tilde{D}_{N-s}\left(\tilde{\mathbf{q}}_{1}^{*}, \ldots, \tilde{\mathbf{q}}_{N-s}^{*}\right) \times\right. \\
& \left.\times \prod_{i=1}^{N-s}\left(\tilde{f}_{s}\left(\tilde{\mathbf{q}}_{1}, \ldots, \tilde{\mathbf{q}}_{s} ; \tilde{\mathbf{q}}_{i}^{*}\right)+1\right)\right] \tilde{n}_{s}\left(\tilde{\mathbf{q}}_{1}, \ldots, \tilde{\mathbf{q}}_{s}\right)
\end{aligned}
$$

with

$$
\begin{aligned}
\tilde{f}_{s}\left(\tilde{\mathbf{q}}_{1}, \ldots, \tilde{\mathbf{q}}_{s} ; \tilde{\mathbf{y}}\right) & \stackrel{\text { def }}{=} \prod_{i=1}^{s} \exp \left[-\beta \tilde{\Phi}\left(\tilde{\mathbf{q}}_{i}, \tilde{\mathbf{y}}\right)\right]-1, \\
\tilde{n}_{s}\left(\tilde{\mathbf{q}}_{1}, \ldots, \tilde{\mathbf{q}}_{s}\right) & \stackrel{\text { def }}{=} \exp \left[-\beta \sum_{1 \leq i<j \leq s} \tilde{\Phi}\left(\tilde{\mathbf{q}}_{i}, \tilde{\mathbf{q}}_{j}\right)\right] .
\end{aligned}
$$

We come then to the problem of finding an upper bound for the fraction $Q_{N-s} / Q_{N}$ and for the term in square brackets in (53). 
As far as the fraction is concerned, it is shown 4 in [6] that

$$
\begin{aligned}
\frac{Q_{M}}{Q_{M-1}} \geq & \frac{1}{Q_{M-1}} \int_{\tilde{V}} \mathrm{~d}^{3} \tilde{\mathbf{q}}_{1} \ldots \int_{\tilde{V}} \mathrm{~d}^{3} \tilde{\mathbf{q}}_{M-1} \exp \left(-\beta \sum_{1 \leq i<j \leq M-1} \tilde{\Phi}\left(\tilde{\mathbf{q}}_{i}, \tilde{\mathbf{q}}_{j}\right)\right) \times \\
& \times\left[\int_{\tilde{V}} \mathrm{~d}^{3} \tilde{\mathbf{q}}\left(1-\sum_{l=1}^{M-1}\left|e^{-\beta \tilde{\Phi}\left(\tilde{\mathbf{q}}, \tilde{\mathbf{q}}_{l}\right)}-1\right|\right)\right] .
\end{aligned}
$$

Integrating over $\tilde{\mathbf{q}}$, we obtain that the term in square brackets at the r.h.s. of this inequality is bounded from below by

$$
V \frac{\tilde{L}}{L}-(M-1) \sup _{\tilde{\mathbf{q}}_{l} \in \tilde{V}} \int_{\tilde{V}} \mathrm{~d}^{3} \tilde{\mathbf{q}}\left|e^{-\beta \tilde{\Phi}\left(\tilde{\mathbf{q}}, \tilde{\mathbf{q}}_{l}\right)}-1\right| \geq V \frac{\tilde{L}}{L}-(M-1) I,
$$

with $\tilde{L}$ defined by (31) in the statement of Lemma 2. Therefore, if hypothesis (32) holds, one has

$$
\frac{V Q_{M-1}}{Q_{M}} \leq \frac{1}{\tilde{L} / L-\rho I} \leq 2
$$

Thus, we can write

$$
\frac{V^{s} Q_{N-s}}{Q_{N}}=\prod_{i=1}^{s} \frac{V Q_{N-i}}{Q_{N-i+1}} \leq 2^{s}
$$

and get the required upper bound.

As regards the term in square brackets in (53), instead, we expand the product and we get that such a term is equal to

$1+\sum_{k=1}^{N-s}\left(\begin{array}{c}N-s \\ k\end{array}\right) \frac{1}{V^{k}} \int_{\tilde{V}} \mathrm{~d}^{3} \tilde{\mathbf{q}}_{1}^{*} \ldots \int_{\tilde{V}} \mathrm{~d}^{3} \tilde{\mathbf{q}}_{k}^{*} \prod_{i=1}^{k} \tilde{f}_{s}\left(\tilde{\mathbf{q}}_{1}, \ldots, \tilde{\mathbf{q}}_{s} ; \tilde{\mathbf{q}}_{i}^{*}\right) \tilde{F}_{k}^{(N-s)}\left(\tilde{\mathbf{q}}_{1}^{*}, \ldots, \tilde{\mathbf{q}}_{k}^{*}\right)$.

We know an uniform upper bound for $\tilde{F}_{k}^{(N-s)}$, namely,

$$
\sup _{\tilde{\mathbf{q}}_{1} \in \tilde{V}, \ldots, \tilde{\mathbf{q}}_{k} \in \tilde{V}}\left|\tilde{F}_{k}^{(N-s)}\right| \leq \frac{\left(2 e^{2 b+1}\right)^{k}}{1-\exp \left(2 \rho e^{2 b+1} I-1\right)},
$$

which holds in the hypotheses

$$
\frac{V+I}{2 e^{2 b+1}(\tilde{L} / L)(V(\tilde{L} / L)-I)}<1 \quad \text { and } \quad \frac{V}{4 e^{2 b+2}(\tilde{L} / L)(V(\tilde{L} / L)-I)}<1 .
$$

These conditions are certainly satisfied if hypothesis (30) holds. Inequality (55) has been proved 5 by Bogolyubov et al. in work [6], which was dealing

\footnotetext{
${ }^{4}$ See formulas (3.20) and (3.21) in 6].

${ }^{5}$ See the deduction of formula (4.10) in 6].
} 
with pair potentials depending only on the distance between two particles. On the other hand, the hypothesis on the dependence on distance is not crucial, and a proof can be produced in the weaker hypothesis of potentials symmetric under the exchange of $i$ and $j$. Indeed, the only difference from the proof given in [6] would be in the construction of the functions $\nu_{i}$, but we show in Lemma 3 that it is possible to construct such functions in the present case, too. The $\nu_{i}$ are introduced in connection with the symmetrization operator $\pi_{l}$, which acts on the function $f\left(\mathbf{q}_{1}, \ldots, \mathbf{q}_{s}\right)$ through the formula

$$
\pi_{l} f\left(\mathbf{q}_{1}, \mathbf{q}_{2}, \ldots, \mathbf{q}_{l-1}, \mathbf{q}_{l}, \mathbf{q}_{l+1}, \ldots, \mathbf{q}_{s}\right)=f\left(\mathbf{q}_{l}, \mathbf{q}_{2}, \ldots, \mathbf{q}_{l-1}, \mathbf{q}_{1}, \mathbf{q}_{l+1}, \ldots, \mathbf{q}_{s}\right) .
$$

One has the following lemma, whose proof can be found in Appendix C.

Lemma 3 Suppose there exists a positive constant $b$ such that, for all s and $\left(\mathbf{q}_{1}, \ldots, \mathbf{q}_{s}\right)$, the potential $\varphi\left(\mathbf{q}_{i}, \mathbf{q}_{j}\right)=\varphi\left(\mathbf{q}_{j}, \mathbf{q}_{i}\right)$ satisfies

$$
\sum_{1 \leq i<j \leq s} \beta \varphi\left(\mathbf{q}_{i}, \mathbf{q}_{j}\right) \geq-s b
$$

Then, for all $s$, there exist measurable functions $\nu_{i}\left(\mathbf{q}_{1}, \ldots, \mathbf{q}_{s}\right)$, having values in the interval $[0,1]$, and such that

$$
\sum_{i=1}^{s} \nu_{i}\left(\mathbf{q}_{1}, \ldots, \mathbf{q}_{s}\right)=1, \quad \nu_{k}\left(\mathbf{q}_{1}, \ldots, \mathbf{q}_{s}\right)=\pi_{k} \nu_{1}\left(\mathbf{q}_{1}, \ldots, \mathbf{q}_{s}\right)
$$

with the inequality

$$
\beta \sum_{j \neq i} \varphi\left(\mathbf{q}_{i}, \mathbf{q}_{j}\right)>-2 b
$$

holding if $\nu_{i}\left(\mathbf{q}_{1}, \ldots, \mathbf{q}_{s}\right) \neq 0$.

The only difference with respect to the functions used in [6] is that here the functions $\nu_{i}$ are not invariant under the rotation group. This, however, does not affect the proof, because it turns out that the proof given in [6] can be repeated word by word. This gives the upper bound (55).

For what concerns $\tilde{f}_{s}$, we adapt to the present situation the reasoning followed by O. Penrose in work [7], which deals with hard-core potentials, in order to provide an upper bound in this case. Indeed, we notice that, by condition (29), one has

$$
1+\tilde{f}_{s}\left(\tilde{\mathbf{q}}_{1}, \ldots, \tilde{\mathbf{q}}_{s} ; \mathbf{y}\right)=\prod_{i=1}^{s} \exp \left[-\beta \tilde{\Phi}\left(\tilde{\mathbf{q}}_{i}, \tilde{\mathbf{y}}\right)\right] \leq e^{2 s b}
$$


Defining

$$
g_{i} \stackrel{\text { def }}{=} \exp \left[-\beta \tilde{\Phi}\left(\tilde{\mathbf{q}}_{i}, \tilde{\mathbf{y}}\right)\right]-1
$$

and $g_{i \pm}=\max \left(0, \pm g_{i}\right) \geq 0$, we can use the upper bound in (59) and the fact that $g_{i} \geq-1$, in order to prove by induction on $s$ that

$$
1-\sum_{i=1}^{s} g_{i-} \leq \prod_{i=1}^{s}\left(1+g_{i}\right) \leq 1+e^{2 s b} \sum_{i=1}^{s} \frac{g_{i+}}{1+g_{i+}} .
$$

We can use this relation in (59) to obtain

$$
\left|\tilde{f}_{s}\left(\tilde{\mathbf{q}}_{1}, \ldots, \tilde{\mathbf{q}}_{s} ; \mathbf{y}\right)\right| \leq \sum_{i=1}^{s} \max \left(g_{i-}, \frac{e^{2 s b} g_{i+}}{1+g_{i+}}\right) .
$$

Then, the integral over $\mathbf{y}$ of this quantity, for every choice of $\left(\tilde{\mathbf{q}}_{1}, \ldots, \tilde{\mathbf{q}}_{s}\right)$, is smaller than $s I_{s}$, with $I_{s}$ defined by (34) if $s \geq 2$, while the case of $s=1$ is trivial. If one, eventually, recalls that

$$
\left(\begin{array}{c}
N-s \\
k
\end{array}\right)<\frac{N^{k}}{k !}
$$

one can then bound (53) from above by

$$
\tilde{F}_{s}^{(N)}\left(\tilde{\mathbf{q}}_{1}, \ldots, \tilde{\mathbf{q}}_{s}\right) \leq 2^{s} \frac{\exp \left(2 \rho e^{2 b+1} s I_{s}\right)}{1-\exp \left(2 \rho e^{2 b+1} I-1\right)} \tilde{n}_{s}\left(\tilde{\mathbf{q}}_{1}, \ldots, \tilde{\mathbf{q}}_{s}\right) .
$$

Observing that, for densities lower than $1 / 4 e I$, the denominator of the fraction in the r.h.s. is larger than $(\sqrt{e}-1) / \sqrt{e}$, the thesis is finally proved by going back to the initial coordinates and using relation (52).

Q.E.D.

\section{Proof of Lemma 3}

We show how to construct the functions $\nu_{i}$ having the properties required in the lemma.

First, we fix a value for $s$ and consider the subsets $A_{i}$ of the configuration space $X$, which are defined, for all $i \leq s$, by

$$
A_{i} \stackrel{\text { def }}{=}\left\{x: \beta \sum_{j \neq i} \varphi\left(\mathbf{q}_{i}, \mathbf{q}_{j}\right)>-2 b\right\}
$$


where $x \stackrel{\text { def }}{=}\left(\mathbf{q}_{1}, \ldots, \mathbf{q}_{s}\right)$. We observe now that, due to condition (156) $)$, one has

$$
\bigcup_{i} A_{i}=X
$$

We can thus choose the function $\nu_{1}(x)$ as

$$
\nu_{1}(x)=N(x) \chi_{A_{1}}(x),
$$

where $\chi_{A}$ is the charachteristic function of the set $A$ and the function $N(x)$, which is introduced to normalize the sum, takes the value $1 / n$ if $k$ belongs to $n$ sets $A_{i}$ but not to $n+1$ of them. Obviously, $N(x)$ takes values in the set $\{1,1 / 2, \ldots, 1 / s\}$.

Then, if we construct $\nu_{k}$ by $\nu_{k}=\pi_{k} \nu_{1}$, it is clear that the functions $\nu_{i}$ satisfy condition (58), because $\pi_{k} \chi_{A_{1}}=\chi_{A_{k}}$ and because of the definition of $A_{i}$. On the other hand, condition (57) holds, too, because $\pi_{k} N(x)=N(x)$. Indeed, the belonging of $x$ to sets different from $A_{1}$ and $A_{k}$ is not affected by the action of $\pi_{k}$, while $\pi_{k} x$ belongs to $A_{1}$ if and only if $x$ belongs to $A_{k}$ and $\pi_{k} x$ belongs to $A_{k}$ if and only if $x$ belongs to $A_{1}$. Thus, the number of sets to which $x$ belongs does not change under the action of $\pi_{k}$, and this completes the proof.

Q.E.D.

\section{References}

[1] C. Cercignani, The Boltzmann equation and its applications (SpringerVerlag, New York 1988).

[2] G. Gallavotti, Statistical Mechanics (Springer-Verlag, Berlin 2000).

[3] R. Kubo, Journal of the Physical Society of Japan 12, (1957) 570.

[4] A. Carati, Journal of Statistical Physics 128, (2007) 1057.

[5] D. Ruelle, Statistical Mechanics, Rigorous Results(Benjamin, New York 1969).

[6] N. N. Bogolyubov, B. I. Khatset, D. Ya. Petrina, Ukrainian Journal of Physics, 53, Special Issue, (2008) 168, available at http://www.ujp.bitp.kiev.ua/files/papers/53SI34p.pdf; Russian original in Teoreticheskaya i Matematicheskaya Fizika, 1:2, (1969) 251. 
[7] O. Penrose, Journal of Mathematical Physics 6, (1963) 1312.

[8] R. A. Minlos, Introduction to Mathematical Statistical Physics (American Mathematical Society, Providence 2000).

[9] B. O. Koopman, Proceedings of the National Academy of Sciences 17, (1931) 315.

[10] R. E. Showalter, Hilbert Space Methods for Partial Differential Equations (Pitman, London 1977).

[11] O. E. Lanford, in Statistical Mechanics and Mathematical Problems (Springer-Verlag, Berlin 1973) 1. 